\title{
Estresse por calor na Atenção Primária à Saúde: uma revisão clínica
}

\section{Heat stress in Primary Health Care: A clinical review \\ Estrés por calor en la Atención Primaria a la Salud: una revisión clínica}

\author{
Mayara Floss ${ }^{1,2}$, Enrique Falceto Barros ${ }^{1,3}$ (D) \\ ${ }^{1}$ Working Party on the Environment da World Organization of Family Doctors (WONCA). \\ ${ }^{2}$ Grupo Hospitalar Conceição (GHC). Porto Alegre, RS, Brasil. \\ ${ }^{3}$ Clínica da Família Teewald (ESF/SUS). Santa Maria do Herval, RS, Brasil.
}

\section{Resumo}

Introdução: A exposição nociva ao calor ganha mais relevância com a progressão do aquecimento global antropogênico e a Atenção Primária à Saúde (APS) tem um papel crescente nesse cenário. No Brasil, as ondas de calor entre 2014 e 2015 duraram mais tempo que nos anos prévios, além disso, entre 2000-2015 a associação entre temperatura e hospitalizações variou de acordo com a duração da exposição ao calor. Nesse contexto, o objetivo desta revisão é realizar uma atualização sobre manejo clínico de patologias relacionadas ao calor na APS. Metodologia: Realizou-se a busca na base de dados ACCESSS, que utiliza a pirâmide 5.0 da assistência à saúde baseada em evidências. Foram identificados 103 sumários sintetizados para referência clínica com as palavras "Heat stress", "Heat Stroke", "Heat Wave" e "Heat Exhaustion", mas apenas três entravam no escopo deste estudo. Resultados e Discussão: O estresse pelo calor é uma condição comum, negligenciada e evitável que afeta diversos pacientes, iniciando-se com uma má adaptação ao calor que se não for corrigida pode gerar uma cascata de eventos inflamatórios. O estresse pelo calor é caracterizado por sintomas inespecíficos, como mal-estar, cefaleia e náusea. O tratamento envolve o resfriamento do paciente e monitoramento, garantindo hidratação adequada. A exaustão pelo calor, se não tratada, pode evoluir para insolação, uma doença grave que pode levar ao coma e morte, envolvendo disfunção do sistema nervoso central - necessitando de um tratamento mais agressivo além do resfriamento.

Palavras-chave: Transtornos de Estresse por Calor; Temperatura Alta; Onda de Calor; Exaustão por Calor

Como citar: Floss M, Barros EF. Estresse por calor na Atenção Primária à Saúde: uma revisão clínica. Rev Bras Med Fam Comunidade. 2020;15(42): 1948. https://doi.org/10.5712/rbmfc15(42)1948
Autor correspondente:

Mayara Floss.

E-mail: mayarafloss@hotmail.com

Fonte de financiamento:

declaram não haver.

Parecer CEP:

não se aplica.

Procedência e revisão por pares:

Não encomendado;

revisão por pares externa.

Recebido em: 07/01/2019.

Aprovado em: 27/08/2019. 


\begin{abstract}
Introduction: The nocive exposure to heat gets more attention with anthropogenic global warming, and Primary Health Care (PHC) has a growing role in this scenario. In Brazil heat waves between 2014 and 2015 lasted longer than in previous years. Further, in addition between $2000-2015$ the association between temperature and hospitalizations varied according to the duration of heat exposure. Therefore, the aim of this review is to perform an update on clinical management of heat related pathologies in PHC. Methodology: The ACCESSS database was searched using the evidence-based health care pyramid 5.0, where we identified 103 synthesized summaries for clinical reference with words "Heat stress", "Heat Stroke", "Heat Wave" and "Heat Exhaustion", but only three fell within the scope of this study. Results and Discussion: Heat stress is a common, neglected and preventable condition that affects several patients, it starts with a poor adaptation to heat that if it is not adjusted it can generate a cascade of inflammatory events. Heat stress is characterized by nonspecific symptoms such as malaise, headache and nausea. The treatment involves patient monitoring and cooling, ensuring adequate hydration. Heat exhaustion, if untreated, can progress to heatstroke, a serious illness that can lead to coma and death, involving central nervous system dysfunction - requiring more aggressive treatment than cooling.
\end{abstract}

Keywords: Heat Stress Disorders; Hot Temperature; Heat Wave (Meteorology); Heat Exhaustion

\title{
Resumen
}

Introducción: La exposición nociva al calor gana más destaque con la progresión del calentamiento global antropogénico, y la Atención Primaria a la Salud tienen un papiel cresciente en este escenario. En Brasil las olas de calor entre 2014 y 2015 duraron más tiempo que en los años previos, además entre 2000-2015 la asociación entre temperatura y hospitalizaciones ha variado de acuerdo con la duración de la exposición al calor. En este contexto, el objetivo de esta revisión es realizar una actualización sobre manejo clínico de patologías relacionadas al calor en la APS Metodología: Se realizó la búsqueda en la base de datos ACCESSS, que utiliza la pirámide 5.0 de la asistencia a la salud basada en evidencias. Se han identificado 103 sumarios sintetizados para referencia clínica con las palabras "Heat stress", "Heat Stroke", "Heat Wave" y "Heat Exhaustion", pero sólo tres son considerados en el ámbito de este estudio. Resultados y Discusión: El estrés por el calor es una condición común, descuidada y evitable que afecta a varios pacientes, iniciándose con una mala adaptación al calor que si no se corrige puede generar una cascada de eventos inflamatorios. El estrés por el calor se caracteriza por síntomas inespecíficos, como malestar, cefalea y náuseas. El tratamiento implica el enfriamiento del paciente y el monitoreo, garantizando la hidratación adecuada. El agotamiento por el calor, si no se trata, puede evolucionar hacia la insolación, una enfermedad grave que puede llevar al coma y a la muerte, involucrando disfunción del sistema nervioso central - necesitando un tratamiento más agresivo además del enfriamiento.

Palabras clave: Trastornos de Estrés por Calor; Calor; Ola de Calor; Agotamiento por Calor

\section{Introdução}

A questão da exposição nociva ao calor ganha mais destaque com a progressão do aquecimento global antropogênico. ${ }^{1}$ Ainda há pouca familiaridade médica com a morbimortalidade pelo estresse ao calor, mas nesse novo contexto os médicos de família e comunidade (MFCs) devem estar atentos para integrar essa questão na sua formação, especialmente dentro de uma perspectiva mais holística de saúde planetária. ${ }^{2}$

Em 2019, a WONCA lançou a Conclamação para a ação dos médicos de família de todo o mundo, com ações necessárias para mitigar os efeitos das mudanças climáticas, incluindo orientações para pacientes e profissionais e colocando o MFC como um ator central em suas comunidades. ${ }^{3,4}$ Os médicos de família estão na linha de frente da proteção à saúde, e é importante reconhecer as interligações entre a mudança ambiental e os impactos emergentes na saúde. ${ }^{3,4}$

Todos os anos, milhões de pessoas são expostas aos perigos dos extremos de calor e ondas de calor que podem ser letais. Em 2017 aconteceu um aumento de mais de 18 milhões de exposições a ondas de calor de pessoas vulneráveis em relação a 2016. ${ }^{1}$ As ilhas de calor, criadas em centros urbanos densamente povoados, com telhados escuros, asfalto e outras infraestruturas, tendem a concentrar o calor solar e impedir sua dispersão. 5,6

No relatório do Lancet Countdown Brasileiro constatou-se que as ondas de calor em 2014 e 2015 no Brasil duraram mais tempo que nos anos prévios. O aumento da temperatura e as ondas de calor afetam 
mais os idosos, gestantes, crianças, atletas e pessoas com doenças crônicas..$^{5,7}$ No Brasil entre 2000-2015 a associação entre temperatura e hospitalizações variou conforme a duração da exposição ao calor, mas também por sexo, idade e região. ${ }^{5}$

Se um paciente apresenta estresse por calor, o diagnóstico rápido e o resfriamento efetivo são cruciais para evitar a evolução para a insolação, que pode evoluir para lesão irreversível ou morte - nesse sentido, o manejo inicial no contexto da APS é essencial. ${ }^{7}$ Esta revisão apresenta aspectos do diagnóstico de estresse por calor e insolação, manejo e prevenção.

\section{Métodos}

Esta revisão de literatura foi desenvolvida por busca de artigos indexados na base de dados ACCESSSS.

\section{Estratégia de Busca}

A base de dados ACCESSSS foi escolhida por ser mais vantajosa como uma busca federada que realiza a busca simultânea em várias bases de dados ${ }^{8}$ incluindo, por exemplo, sumários de textos clínicos como Best Practice (BMJ), Dynamed e EBM Guidelines; Revisões sistemáticas, Estudos Originais e Revisões Sistemáticas. A base de dados ACCESSSS utiliza a pirâmide 5.0 da assistência à saúde baseada em evidências e é uma forma de organizar o conhecimento hierarquicamente com as informações voltadas para a assistência à saúde. ${ }^{8}$

Os critérios de inclusão para esta revisão clínica foram: estudos publicados a partir de 2010, com a característica de serem revisões sistemáticas e diretrizes clínicas sobre estresse por calor (e palavras-chave selecionadas), ser em inglês, português ou espanhol. Pesquisas originais foram buscadas e utilizadas em casos de dúvidas em relação a alguma informação das diretrizes clínicas e revisões sistemáticas. Os critérios de exclusão foram: não ter o estresse por calor como tema da pesquisa, ser um estudo anterior a 2010, e ser em outra língua que não as selecionadas. A pesquisa foi realizada entre março e abril de 2019.

Utilizaram-se os descritores com as palavras "Heat stress", "Heat Stroke", "Heat Wave" e "Heat Exhaustion" (Tabela 1). Foram selecionados artigos de sumários sintetizados para referência clínica de três textos para inclusão neste artigo, caracterizando uma revisão clínica, desta forma, não foram utilizados estudos originais.

Tabela 1. Palavras-chave pesquisadas na base de dados ACCESSS.

\begin{tabular}{|c|c|c|c|c|c|}
\hline \multirow{2}{*}{ Termo } & \multicolumn{5}{|c|}{$\begin{array}{l}\text { Resultados de artigos conforme pirâmide } 5.0 \text { da assistência à saúde baseada em evidências da } \\
\text { base de dados ACCESSSS }\end{array}$} \\
\hline & Sistemas & $\begin{array}{l}\text { Sumários sintetizados } \\
\text { para referência clínica }\end{array}$ & $\begin{array}{l}\text { Revisões } \\
\text { Sistemáticas }\end{array}$ & Estudos originais & Total \\
\hline Heat Stress & 1 & 70 & 3 & 1 & 75 \\
\hline Heat Stroke & 0 & 80 & 0 & 2 & 82 \\
\hline Total de artigos & 1 & 216 & 3 & 8 & 228 \\
\hline Total sem repetições & 1 & 103 & 0 & 3 & \\
\hline Artigos incluídos & 0 & $3^{9-11}$ & 0 & 0 & \\
\hline
\end{tabular}


Adicionalmente, foram pesquisadas revisões sistemáticas na base de dados LILACS com os termos "heat stress", "heat exhaustion" e "heat stroke", sendo encontrado um artigo que não entrava no escopo deste estudo. Ademais, alguns artigos que não são revisões sistemáticas foram utilizados conforme a necessidade para esclarecer dúvidas dos autores em relação aos termos e condutas. ${ }^{12-16}$ Por último, utilizaram-se recomendações de políticas de saúde como gray literature para complementar esta revisão clínica. ${ }^{3-5}$

\section{O estresse por calor e insolação}

O estresse por calor e a insolação ocorrem quando o calor sobrecarrega a resposta termorreguladora e a homeostase do corpo. Extremos de temperatura e umidade tornam a dissipação de calor menos eficiente e podem levar ao estresse por calor e insolação. ${ }^{9}$

O estresse ou exaustão pelo calor é uma doença leve a moderada devido à perda de sal e água durante a exposição ao calor e a falha de mecanismos termorregulatórios fisiológicos para compensar o aumento da temperatura corporal devido a temperaturas elevadas, esforço físico ou metabolismo. Já a insolação é caracterizada por uma temperatura corporal central $\geq 40^{\circ} \mathrm{C}$ com disfunção do sistema nervoso central. Pode progredir para falha do sistema de múltiplos órgãos. ${ }^{9-11}$

O MFC deve sempre suspeitar de insolação, principalmente após atividade física em ambiente vulnerável ao calor excessivo, quando o paciente apresenta pele quente, alteração da consciência, hipotensão, hiperventilação, náusea ou diarreia. Pode haver sudorese excessiva, porém pode existir uma pele quente e "seca" quando desidratação se desenvolve. ${ }^{10,11}$ As câimbras de calor são contrações musculares involuntárias associadas ou não ao exercício (geralmente nas extremidades inferiores). ${ }^{10}$ Câimbras pelo calor excessivo geralmente envolvem vários grupos musculares, diferentemente das câimbras relacionadas aos esportes, que ocorrem geralmente em um único músculo/grupo muscular. ${ }^{12}$

Os fatores de risco para qualquer doença relacionada ao calor incluem:9-12

- Fatores demográficos/ Baixo status socioeconômico;

- Idade (crianças e idosos), principalmente pacientes acamados;

- Sistema de ar condicionado inadequado ou inexistente;

- Atividades ocupacionais ou recreativas que exijam exercícios extenuantes em ambientes quentes e/ou úmidos (por exemplo, agricultores, trabalhadores ribeirinhos ou da floresta, atletas, bombeiros, militares), quanto maior a umidade relativa do ar, maior a vulnerabilidade ao calor;

- Doença mental.

Substâncias podem predispor ao risco de insolação (por exemplo, diuréticos, anti-hipertensivos, anticolinérgicos, antidepressivos tricíclicos, anfetaminas e outros). (Quadro 1)

Algumas medicações podem afetar o mecanismo de adaptação ao calor como: antiadrenérgicos e betabloqueadores podem diminuir o débito cardíaco; anticolinérgicos e antidepressivos tricíclicos podem alterar a eliminação de suor e os antidepressivos tricíclicos podem alterar a termorregulação central; anti-histamínicos e agentes antiparkinsonianos podem causar inibição do mecanismo da sudorese; antipsicóticos podem inibir o mecanismo de sudorese e causar a síndrome Neuroléptica Maligna, sendo que o calor pode agravar este sintoma; simpaticomiméticos podem causar desidratação e hiponatremia., ${ }^{73}$ 
Quadro 1. Substâncias que podem estar relacionadas à piora dos sintomas.

Substância que podem agravar os sintomas do estresse por calor e insolação

Álcool

Anfetaminas

Antiadrenérgicos e Betabloqueadores

Anticolinérgicos

Antidepressivos

Anti-histamínicos

Agentes antiparkinsonianos

Antipsicóticos

Cocaína

Diuréticos

Simpaticomiméticos

Outras classes (inibidores da colinesterase, antiarrítmicos, bloqueadores do canal de cálcio)

Ainda, o uso de drogas sintéticas como 3,4-metilenodioximetanfetamina (MDMA) conhecido como "ecstasy" pode confundir os sintomas com a overdose clássica e postergar o diagnóstico por insolação. Outras drogas, como álcool, também são um fator de risco emergente e importante para insolação. ${ }^{13}$

Pacientes com função cognitiva prejudicada (por exemplo, demência, doença de Parkinson) podem ser menos capazes de reconhecer e responder ao calor excessivo. ${ }^{15}$

\section{Avaliação e diagnóstico}

O diagnóstico de doenças relacionadas ao calor é feito clinicamente com base em:-11

- História recente de exposição a ambientes quentes e/ou atividade física;

- Temperatura corporal central (medição retal preferida);

- Insolação definida por temperatura corporal central $\geq 40{ }^{\circ} \mathrm{C}$ com disfunção do sistema nervoso central;

- Outras doenças relacionadas ao calor associadas à temperatura $<40^{\circ} \mathrm{C}$;

- Síncope térmica não requer elevação da temperatura central para fazer diagnóstico;

- Temperatura corporal normal com câimbras pelo calor, edema por calor e erupções cutâneas;

- Sinais e sintomas característicos, como alteração do estado mental, níveis de fadiga, náusea/ vômito e aumento da frequência cardíaca;

- Resolução dos sintomas após o tratamento.

O MFC deve realizar o diagnóstico diferencial da insolação com situações relacionadas à hipertermia com alteração do estado mental como: delirium orgânico, sepse, meningite, cetoacidose diabética, hipertireoidismo, feocromocitoma, síndrome serotoninérgica, infecções do sistema nervoso central, hipertermia maligna, crise tireotóxica, síndrome neuroléptica maligna, toxicidade por drogas, delirium tremens. $^{9-12}$

Se houver suspeita de insolação, que é uma emergência médica, o MFC deve encaminhar o paciente para um centro de atenção secundário ou terciário com infraestrutura de suporte avançado e 
para a realização de exames complementares para descartar outras condições e avaliar a morbidade por insolação. O uso racional dos exames e a observação clínica devem ser levados em consideração (veja a seção manejo) aliados à prevenção quaternária. Em pacientes com suspeita de insolação devem ser considerados os exames complementares: ${ }^{9-11}$

- Eletrólitos séricos;

- Hemograma completo, painel metabólico com enzimas hepáticas e lactato;

- Função renal com exame de urina;

- Creatina fosfoquinase (CPK), a CPK >100,000 U/MI é comum e CPK> 5 vezes o limite superior do normal indica possível rabdomiólise e progressão para lesão renal aguda;

- Parâmetros de coagulação para avaliar a coagulação intravascular disseminada (CIVD) ou a síndrome da resposta inflamatória sistêmica (SIRS);

- Radiografia de tórax;

- Gasometria arterial;

- Toxicologia (em caso de abuso de substâncias);

- Função da tireoide;

- Glicose.

Como as manifestações clínicas do estresse por calor são inespecíficas, incluindo alteração do estado mental, entre outras anormalidades do sistema nervoso central, deve-se pensar em possíveis diagnósticos diferenciais, podendo ser necessário considerar outros exames: ${ }^{9}$

- Tomografia computadorizada (TC) da cabeça se houver sinais de déficits neurológicos ou evidência de trauma, ou se os sintomas não se resolverem com tratamento adequado;

- Punção lombar se houver suspeita de infecção do sistema nervoso;

- Cultura de sangue deve ser considerada se sepse for uma causa do aumento da temperatura corporal.

Ainda para diagnóstico diferencial, algumas recomendações incluem: ${ }^{7}$

- Eletrocardiograma de 12 derivações;

- Enzimas cardíacas.

Em adultos com insolação internados no hospital, as funções hepática, renal e de coagulação devem ser monitoradas por 48 horas após a admissão e tratamento (BMJ). ${ }^{9}$

Certamente, estes tópicos não são tão sensíveis à APS, porém é essencial o médico de família e comunidade entender a dimensão dos impactos na saúde, inclusive para referenciar o paciente para o centro mais adequado. 


\section{Manejo}

O diagnóstico preciso do estresse por calor ou insolação em adultos baseia-se na quantificação correta da temperatura central. Pacientes com suspeita de insolação devem ser rapidamente avaliados usando Advanced Trauma e Life Support (ATLS). ${ }^{10,11} \mathrm{O}$ resfriamento precoce (imersão em água com gelo) reduz a mortalidade e a morbidade e deve ser iniciado sem demora. ${ }^{9-11}$ É importante para tanto que a Atenção Primária tenha um plano de cuidado/ação para ondas de calor, inclusive com a preparação de bolsas de gelo, ambientes frescos e/ou climatizados. ${ }^{3}$ Quando disponível, deve-se considerar administrar infusão intravenosa de soro fisiológico em temperatura ambiente ou gelado. ${ }^{8,12}$ Deve-se estar atento que os antipiréticos não são eficazes no tratamento da hipertermia causada por insolação e, por isso, contraindicados.

No CID 10 os códigos relacionados ao estresse por calor estão agrupados no item T67.0 "Golpe de calor e insolação". ${ }^{10}$ Os objetivos do tratamento são o resfriamento rápido e o suporte para os órgãos em sofrimento. A urgência e o nível de tratamento são ditados pela gravidade da doença (Figura 1).

Para casos de estresse por calor leve:9-11

- Observação em ambiente fresco/arejado e a hidratação pode ser apropriada para jovens adultos saudáveis com exame físico normal;

- O resfriamento pode incluir a movimentação para uma área mais fria próxima, removendo o máximo de roupas possível ou o resfriamento ativo (como áreas ventiladas ou aplicação de material frio ao corpo);

- Hidratação para restabelecer o volume circulante e promover a transpiração.

Para sintomas moderados a graves (como insolação ou estresse por calor grave):

- Comece o resfriamento assim que a via aérea, a respiração e a circulação tiverem sido estabilizadas;

- A imersão em água fria é o método de resfriamento preferido, porém deve-se estar atento para o fato do efeito de queda contínua da temperatura central mesmo após o paciente ser removido da imersão. Para prevenir a hipotermia iatrogênica, os pacientes devem ser removidos do banho de gelo quando sua temperatura central atingir $38,3^{\circ} \mathrm{C}$;

- Em atletas, a imersão em água fria está associada à mais rápida taxa de resfriamento e à menor morbidade e mortalidade;

- Considere toalhas/lençóis encharcados de água gelada combinados com bolsas de gelo na cabeça, região inguinal, axilas e extremidades quando a imersão em água não estiver disponível;

- Administrar fluidos por via oral e/ou intravenosa (a via é determinada pela capacidade do paciente de tolerar líquidos orais) para restabelecer o volume circulante e promover a transpiração; 


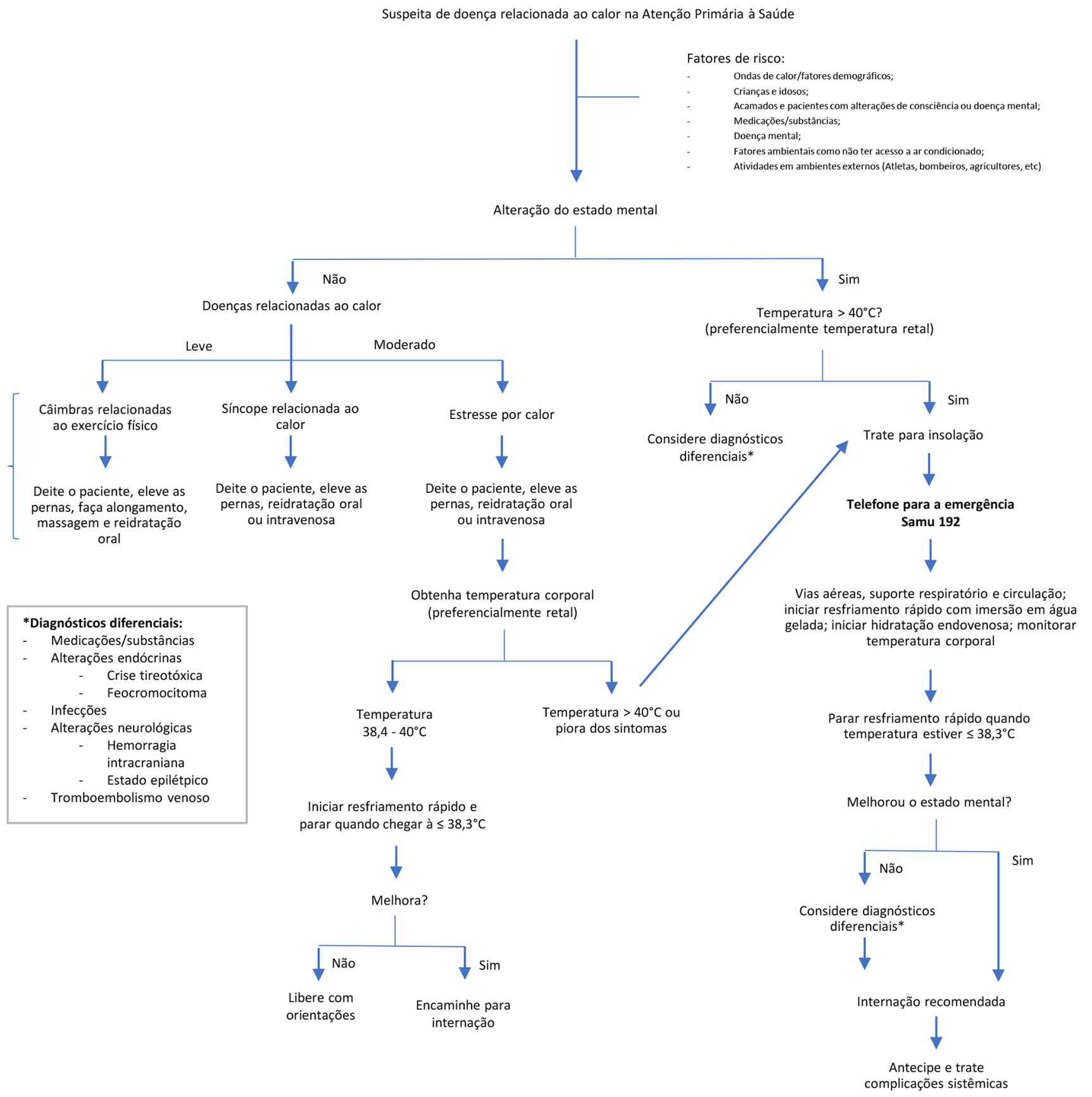

Figura 1. Fluxograma para tratamento de doenças relacionadas ao calor na APS. Adaptado de: Gauer R, Meyers B. Heat-Related IIInesses. Am Fam Physician. 2019;99(8):482-9. ${ }^{12}$

- Para pacientes com sintomas moderados (dor de cabeça, náusea ou vômito) por hiponatremia, considerar a administração de sódio hipertônico somente se os níveis séricos ou plasmáticos de sódio estiverem criticamente baixos (<120 mEq/L);

- Para os doentes com sintomas graves (confusão ou convulsões) por hiponatremia, considerar dar solução salina hipertônica (tal como 3\% de solução salina dada como $100 \mathrm{~mL}$ de bolus até três vezes, 10 minutos de intervalo) e transporte para o hospital;

- Para pacientes com insolação, também monitore os níveis de pressão arterial, débito urinário, pressão venosa central, lactato e creatinofosfoquinase (CPK). 
Tratamento adicional para doenças específicas incluem: ${ }^{11}$

- Para exaustão pelo calor por esforço, elevar as pernas;

- Para câimbras de calor, alongamento e massagem;

- Para erupção cutânea, considere tratamento para prurido associado com medicações tópicas e/ ou compressas frias;

- Se houver suspeita de hipertermia devido ao uso de medicamentos, interrompa a medicação, se possível.

Se os sintomas não se resolverem com o tratamento, considere outras condições que se apresentem com aumento de temperatura e alteração do Sistema Nervoso Central. ${ }^{9-11}$ Complicações como estresse respiratório, choque, insuficiência renal aguda, convulsões, rabdomiólise, CIVD e falência múltipla de órgãos podem acontecer se o tratamento e o resfriamento não forem iniciados em tempo. ${ }^{8}$

O prognóstico depende de quanto tempo o paciente ficou exposto ao calor e às complicações associadas. Pacientes com histórico de insolação têm mais chances de um novo evento. ${ }^{8}$

\section{Prevenção e ações para mitigar os efeitos das ondas de calor}

A inclusão no sistema de vigilância epidemiológica no Sistema de Informação de Agravos de Notificação (SINAN) de doenças relacionadas ao calor é uma ação que pode ser utilizada para planejamento de áreas mais afetadas, ${ }^{5}$ bem como investir em um urbanismo sustentável para diminuir o efeito de ilha de calor, principalmente investindo em áreas verdes. ${ }^{1,3,9} \mathrm{~A}$ integração no currículo dos profissionais de saúde bem como formação continuada são essenciais para lidar com o impacto das ondas de calor e para o reconhecimento dos sintomas..$^{5,9}$

Orientações comunitárias e individuais são aspectos chave para a prevenção primária dos eventos relacionados ao calor. A equipe da APS deve estar ativamente envolvida para:9,13,16

- Educar os pacientes e aumentar a consciência sobre os fatores de risco, sinais, sintomas e tratamento do estresse por calor, tanto para pacientes, cuidadores quanto profissionais de saúde; ${ }^{9,13}$

- Auxiliar o paciente a compreender o seu próprio risco e as ações preventivas que podem tomar para reduzir os riscos durante eventos de calor extremos; 9,13

- Informar a comunidade sobre os riscos e perigos do calor. ${ }^{9}$

Em relação à prevenção de recorrência, deve-se estar atento e realizar recomendações para pacientes que já tiveram um evento de insolação pelo risco mais elevado de um novo evento, bem como pacientes com alto risco como atletas, idosos, crianças e pacientes com doenças crônicas. ${ }^{9}$ 
Por último, vale ressaltar que parte significativa dos estudos refletem a perspectiva da atenção secundária e terciária, portanto, os efeitos nocivos da exposição a extremos de calor merecem ser melhor estudados no contexto da APS. Embora os desafios ambientais globais como as ondas de calor e o estresse por calor representem enormes riscos para a saúde humana, eles também podem oferecer oportunidades para o fortalecimento da APS e sinergias intersetoriais. ${ }^{3}$

\section{Contribuição dos autores}

Concepção e/ou delineamento do estudo: MF, ECF. Aquisição, análise ou interpretação dos dados: MF, ECF. Redação preliminar: MF, ECF. Revisão crítica da versão preliminar: MF, ECF. Todos os autores aprovaram a versão final e concordaram com prestar contas sobre todos os aspectos do trabalho.

\section{Conflito de interesses}

Declaram não haver.

\section{Referências}

1. Watts N, Ammann M, Arnell N, Ayeb-Karlsson S, Belesova K, Berry H, et al. The 2018 report of the Lancet Countdown on health and climate change: shaping the health of nations for centuries to come. Lancet [Internet]. 2018; [acesso 2019 Abr 28]; 392(10163):2479-514. https://doi.org/10.1016/S0140-6736(18)32594-7

2. Xie E, de Barros EF, Abelsohn A, Stein AT, Haines A. Challenges and opportunities in planetary health for primary care providers. Lancet Planetary Health. 2018;2(5):e185-e187. https://doi.org/10.1016/S2542-5196(18)30055-X

3. WONCA. Declaration calling Family Doctors of the world to act on Planetary Health [Internet]; 2019; [acesso 2019 Abr 28]. Disponível em: http://www.globalfamilydoctor.com/Decplanethealth

4. Floss M, Barros E. Saúde planetária: conclamação para a ação dos médicos de família de todo o mundo. Rev Bras Med Fam Comunidade [Internet]. 2019 Mar 1; [acesso 2019 Abr 28];14(41):1992. https://doi.org/10.5712/rbmfc14(41)1992

5. Floss M, Barros E, Bressel M, Hacon S, Stein A, Sirena S, et al. Lancet Countdown 2018 Report: Briefing for Brazilian Policymakers Lancet Countdown [Internet]. 2018; [acesso 2019 Abr 28]; 1:19. Disponível em: http://www.lancetcountdown.org/media/1417/2018-lancet-countdown-policy-brief-brazil.pdf

6. Hoag H. How cities can beat the heat. Nature [Internet]. 2015; [acesso 2019 Abr 28]; 524(7566):402-4. https://doi.org/10.1038/524402a

7. Glazer JL. Management of heatstroke and heat exhaustion. Am Fam Physician [Internet]. 2005; [acesso 2019 Abr 28]; 71(11):2133-40. Disponivel em: https://www.aafp.org/afp/2005/0601/p2133.html

8. Fontenelle LF, Brandão DJ. Uma proposta metodológica para a elaboração de revisões clínicas. Rev Bras Med Fam Comunidade [Internet]. 2018; [acesso 2019 Abr 12];13(40):1-10. https://doi.org/10.5712/rbmfc13(40)1871

9. Glazer JL. Heat Stroke. London: BMJ [Internet]; 2018; [acesso 2019 Abr 28]. Disponível em: https://bestpractice.bmj.com/topics/en-us/849

10. Heat-related illnesses. Dynamed Plus. Ipswich (MA): EBSCO Information Services [Internet]; 2016; [acesso 2019 Abr 28]. Disponível em: http://www.dynamed.com/topics/dmp AN T114802

11. Acute heat illnesses [Internet]. EBM Guidelines; 2018 [acesso 2018 Set 13]. Disponível em: https://www.ebm-guidelines.com/dtk/ebmg/ home?id=ebm00377

12. Gauer R, Meyers B. Heat-Related IIInesses. Am Fam Physician [Internet]. 2019; [acesso 2019 out 01]; 99(8):482-9. Disponível em: https:// www.ncbi.nlm.nih.gov/pubmed/30990296?dopt=Abstract 
13. Health Canada. Extreme Heat Events Guidelines: Technical Guide for Health Care Workers. In: Water AaCCB, ed. Extreme Heat Events Guidelines: Technical Guide for Health Care Workers [Internet]. Ottawa: Health Canada; 2011; [acesso 2019 out 01]; p. 149. Disponível em: https://www.canada.ca/en/health-canada/services/environmental-workplace-health/reports-publications/climate-change-health/ extreme-heat-events-guidelines-technical-guide-health-care-workers.html\#a7.0

14. Nadesan K, Kumari C, Afiq M. Dancing to death: A case of heat stroke. J Forensic Leg Med. 2017;50:1-5. https://doi.org/10.1016/j. jflm.2017.05.008

15. Bouchama A, Dehbi M, Mohamed G, Matthies F, Shoukri M, Menne B. Prognostic factors in heat wave related deaths: a meta-analysis. Arch Intern Med. 2007;167(20):2170-6. https://doi.org/10.1001/archinte.167.20.ira70009

16. Groot E, Abelsohn A, Moore K. Practical strategies for prevention and treatment of heat-induced illness. Can Fam Physician [Internet]. 2014; [acesso 2019 out 01]; 60(8):729-30, e392-4. Disponível em: https://www.ncbi.nlm.nih.gov/pmc/articles/PMC4131963/?report=classic 